\title{
4.6. Documentación de patrimonio cultural en entornos digitales colaborativos: Wikimedia Commons
}

Jesús Tramullas ${ }^{1]}$

Rubén Ojeda ${ }^{2]}$

\section{GLAM y Open GLAM}

El desarrollo de proyectos que llevan a cabo museos, archivos y bibliotecas en el entorno de Wikipedia y del resto de proyectos asociados se ha convertido en los últimos años en una actividad común, que persigue potenciar y desarrollar la misión de las instituciones de la memoria. Instituciones como Library of Congress, British Library, British Museum, Koninklijke Bibliotheek, Rijksmuseum, METS... han llevado a cabo, o participan actualmente, en diferentes tipos de actividades cuya finalidad es poner a disposición de los ciudadanos diversos tipos de contenidos de patrimonio cultural en la red, de forma que se puedan disfrutar y aprovechar libremente. A estas instituciones cabe añadir un buen número de instituciones culturales más modestas que llevan a cabo actividades de estas características. Dentro de su planificación de actividades y proyectos, la propia Europeana ha establecido una línea directa de colaboración con diferentes grupos nacionales e internacionales de editores de Wikipedia para estas acciones.

El acrónimo GLAM corresponde a las iniciales de Galleries, Libraries, Archives and Museums, y bajo el mismo se pretende englobar a las instituciones de la memoria que llevan a cabo proyectos de puesta a disposición de la ciudadanía de material cultural resultante de procesos de digitalización (Kapsalis, 2016). Esta apertura supone la capacidad de potenciar las políticas culturales de las instituciones implicadas, al mismo tiempo que abre nuevas vías para poder evaluar los resultados de las mismas (Saorín, 2013:79).

Hace una década, en 2009, la primera GLAM Wiki Conference, celebrada en Canberra, elaboró un conjunto de recomendaciones que deberían guiar a las iniciativas GLAM, en lo concerniente a liberación de contenidos y licencias de uso, aspectos tecnológicos, educación y desarrollo económico (Véase https://meta.wikimedia.org/wiki/GLAM-WIKI_Recommendations/es). Poco después, la Comisión Europea (2011) publicó una recomendación sobre el material cultural digitalizado y puesto en línea, que contiene, entre otras consideraciones, las siguientes:

“5a) garantizando que el material de dominio público siga perteneciendo al dominio público una vez digitalizado;

5b) promoviendo un acceso lo más amplio posible al material digitalizado de dominio público y a la reutilización de este material para fines comerciales y no comerciales;

5c) adoptando medidas para limitar el uso de marcas intrusivas u otras medidas de protección visual que dificulten la utilización del material digitalizado de dominio público."

En consonancia con el contenido de este documento, y las políticas que impulsaba, La Unión Europea puso en marcha y financió hasta 2016 la iniciativa OpenGLAM, coordinada por el Open Knowledge Project, cuya finalidad era construir una cultura global sobre el procomún, y que desarrolló actividades

1] Universidad de Zaragoza, Depto. Ciencias de la Documentación, tramullas@unizar.es

2] Wikimedia España, Coordinador de Programas. rubenojeda@wikimedia.es 
sobre la liberación de contenidos culturales y su reutilización en diferentes campos. Tras la finalización del proyecto, éste ha sido retomado por Open Knowledge Foundation, Wikimedia Foundation y Creative Commons como una comunidad de interés para el soporte y difusión de OpenGLAM.

OpenGLAM engloba a aquellas iniciativas que pretenden la liberación de contenidos culturales digitalizados, con licencias que hagan posible su reutilización, uso creativo y creación de obras derivadas, incluyendo la explotación comercial de los mismos (Terras, 2015). El elemento clave en esta cuestión es la licencia, o licencias, bajo las cuales se publica el contenido digitalizado. En OpenGLAM, la referencia es la Open Definition (Véase http://opendefinition.org/), o Definición de Conocimiento Abierto. Esta definición permite establecer que licencias son compatibles con la misma, y cuales no. Por ejemplo, Creative Commons de Dominio Público (CCO), Atribución (CC-BY), Atribución-Compartir Igual (CC-BY$S A)$, Licencia de Documentación Libre de GNU, Free Art License y licencias Open Data Commons son licencias que hacen posible el aprovechamiento completo del patrimonio cultural digitalizado.

Han sido numerosas las acciones OpenGLAM que se han llevado a cabo en los últimos años. Por citar algunos de los más relevantes en cuanto a su impacto, debe recordarse que en 2011 el Rijksmuseum liberó 208.000 imágenes de alta calidad. Más recientemente, en 2017, el MET de Nueva York puso en dominio público 375.000 imágenes de alta calidad (fig. 1), y en 2018 ha sido el Art Institute of Chicago el que ha depositado en dominio público más de 44.000 imágenes de objetos provenientes de sus colecciones. No cabe duda que existe una relación entre procesos de digitalización del patrimonio cultural e indicadores de innovación y desarrollo en éste y otro ámbitos asociados, como ha sido señalado por Borowiecki y Navarrete (2016).

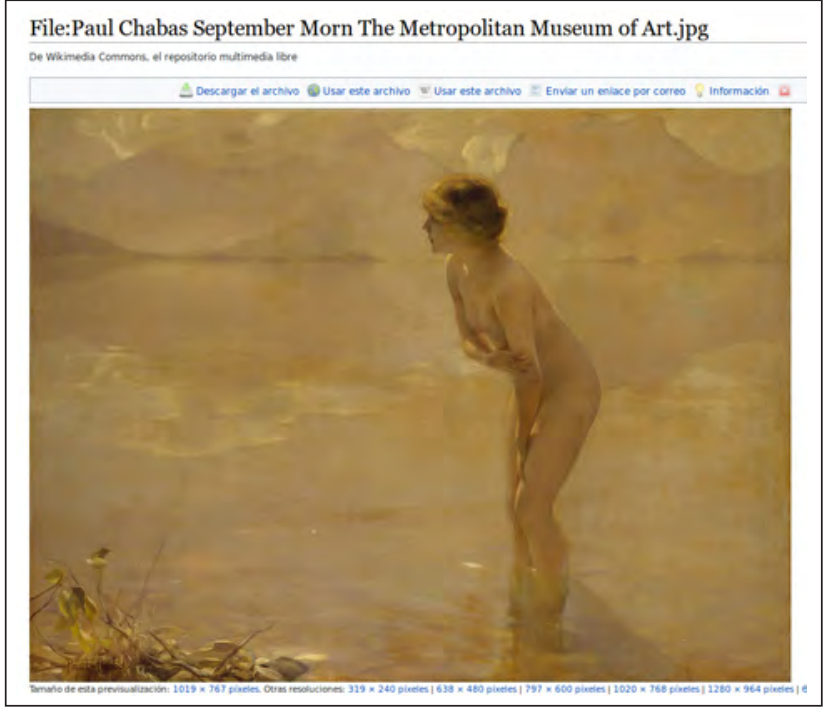

Fig. 1. Digitalización liberada por el MET en Commons (https://commons.wikimedia.org/ wiki/File:Paul_Chabas_September_Morn_ The_Metropolitan_Museum_of_Art.jpg)

\section{GLAM Wiki: Wikimedia Commons}

En correspondencia con lo señalado en el apartado anterior, GLAM wiki es la expresión que se utiliza para las iniciativas que las instituciones GLAM Ilevan a cabo en el contexto de Wikipedia, de Wikimedia Commons y del resto de proyectos asociados. Los objetivos de los ambos actores coinciden en la finalidad de poner al alcance de la comunidad el acceso a cultura, de una forma abierta y reutilizable, y en desarrollar las habilidades y competencias informacionales de los usuarios (Phillips, 2013). Las vías de esta colaboración pueden incluir la realización de actividades de formación de los profesionales y de 
los usuarios, atendiendo a competencias de organización, redacción y referencia de información, las competencias informacionales, la integración con linked open data, la potenciación de los catálogos y colecciones, el desarrollo del acceso abierto, la creación de comunidades de práctica y la integración en procesos de enseñanza/aprendizaje.

Wikimedia Commons, puesto en marcha en 2004, se ha convertido en un repositorio de referencia para la documentación de patrimonio cultural, merced al desarrollo de proyectos de liberación de material digitalizado por parte de instituciones de la memoria. Las características y formas de aprovechamiento del acervo documental de Commons ha sido explicadas por Pastor Sánchez y Saorín (2011). Commons actúa como un repositorio colaborativo en el cual pueden recogerse y describirse, de forma básica, todo tipo de documentos, objetos y materiales multimedia (imágenes estáticas y en movimiento, texto digitalizados, documentos sonoros...), que pueden ser tanto integrados en los contenidos de Wikipedia, en sus diferentes versiones lingüísticas, como utilizados libremente fuera de ella, para la ilustración y enriquecimiento de cualquier otro tipo de documento, sea físico o digital. Gracias a la aportación de las instituciones GLAM y de la comunidad de editores, estos materiales pueden usarse, modificarse y distribuirse libremente, facilitando el acceso y disfrute cultural, la investigación científica, y la creación cultural a través de la reutilización de los contenidos (Tunchs, 2007).

Las instituciones GLAM recogidas en el párrafo final del apartado primero de este texto han integrado los materiales liberados tanto en sus propios catálogo y exposiciones virtuales, como en Wikimedia Commons y en artículos de Wikipedia. Adicionalmente, nuevos estudios demuestran que la integración de contenidos de las instituciones GLAM en Wikipedia y Commons traen como consecuencia un aumento de la visibilidad de las colecciones y de las actividades de las propias instituciones, y se traducen en un mayor número de visitantes y una mejor imagen pública de las mismas (Navarrete y Borowiecki, 2016).

\section{Tipos de actividades en iniciativas GLAM Wiki}

Los proyectos y acciones GLAM wiki que llevan a cabo las instituciones o las comunidades de usuarios suelen adoptar alguna de las formas de organización que se detallan a continuación (Wikimedia España, 2016). Debe tenerse en cuenta que, en proyectos diseñados para un medio o largo alcance, generalmente se adopta un enfoque basado en un proyecto integrado, que suele incorporar la figura del wikipedista residente, y en el marco del cual se llevan a cabo el resto de acciones:

- Proyectos integrados: se trata de proyectos institucionalizados en el marco de una entidad GLAM o un grupo de editores, que se llevan a cabo con una planificación, objetivos y recursos determinados. Suponen un compromiso por parte de la institución GLAM, y pueden tener diferentes alcance y duración, dependiendo de los objetivos fijados para el mismo (Machefert, 2015). Con responsabilidad en el proyecto figura un miembro de la institución con experiencia en Wikipedia o un wikipedista residente.

- Wikipedista residente: la figura del wikipedista residente suele estar asociado a los proyectos integradores delineados en el párrafo anterior. Se trata de un editor experimentado, que actúa como enlace entre la institución y la comunidad de editores, al tiempo que trabaja internamente con el equipo de la institución en los procesos de publicación y difusión de contenidos. También organiza y coordina otras actividades, como concursos o editatones.

- Liberación de contenidos: se trata de acciones puntuales o programadas de liberación de contenido digitalizado proveniente de las colecciones de la institución GLAM que se trate, o resultados de otras actividades llevadas a cabo por al misma. La forma más común es la publicación y difusión bajo licencias de dominio público, de manera que no pongan límites a los usos que puedan hacerse de las mismas, en línea con las recomendaciones de la Comisión Europea ya citadas y con la propuesta de Europeana. 
- Editatones: son eventos de edición de contenido, que pueden celebrarse en una o varias sesiones, en un momento determinado o la largo de un espacio de tiempo dado (Robichaud, 2016). Se organiza tanto para editores como para nuevos usuarios, y se centran en un tema específico. A tal fin se prepara una documentación básica de referencia, que se utiliza para cumplir con el principio de verificabilidad inherente a Wikipedia, así como una lista de artículos o contenidos a desarrollar. En el editatón se cuenta con la presencia de editores experimentados que actúan como guía del resto de participantes.

- Concursos: competiciones entre editores en las que se persigue aumentar el contenido de Wikipedia, Commons, Wikidata y otros proyectos. Se proyectan concursos temáticos, que pueden ser más o menos específicos en cuanto a contenidos cubiertos, y se desarrollan durante un plazo temporal dado. Son numerosos los concursos, de muy diferente tipo y alcance. Sin embargo, es necesario destacar dos de especial importancia para el patrimonio cultural. En primer lugar Wiki Loves Monuments, que es el mayor concurso mundial, por volumen y variedad, de fotografía sobre patrimonio monumental, en todas sus expresiones (Véanse https://www.wikilovesmonuments.org/ y https://www.wikilm.es/), y, en segundo, Wikis Loves Folk, sobre fiestas de interés turístico (Véase https://www.wikilov.es/es/Wiki_ Loves_Folk).

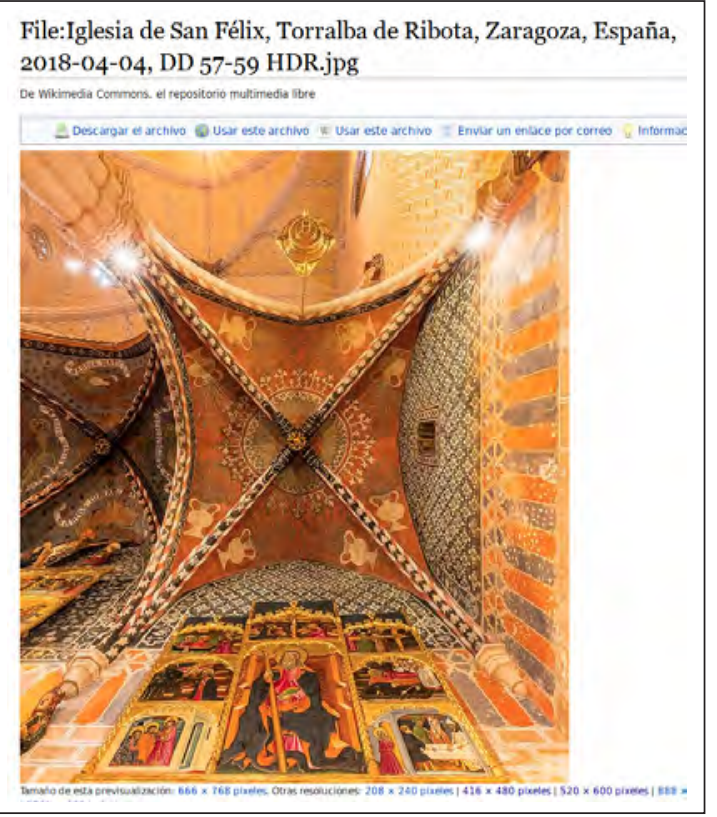

Fig. 2. Fotografía finalista del concurso Wiki Loves Monuments 2018 ( https://commons.wikimedia.org/ wiki/File:Iglesia_de_San_F\%C3\%A9lix,_Torralba_de_ Ribota,_Zaragoza,_Espa\%C3\%B1a,_2018-04-04, DD_57-59_HDR.jpg)

- Wiki Takes: este tipo de acciones se desarrollan sobre un espacio geográfico o arquitéctónico, durante un tiempo dado, que puede oscilar desde media jornada a varios días. En este tipo de actividad un grupo de editores visitan un espacio específico, o lo recorren siguiendo una ruta más o menos establecida, con la finalidad de documentar gráficamente el patrimonio cultural (histórico, etnológico, industrial o de cualquier tipo) existente en el área. Normalmente se buscan áreas de las cuales exista poca o escasa documentación, ya que el objetivo es recoger material gráfico digital (fotografía, vídeo) para asegurar, al menos, su preservación y difusión, y en un momento posterior, poder elaborar nuevos artículos en Wikipedia, e incrementar el acervo documental disponible en Wikimedia Commons. 


\section{Ejemplos de acciones GLAM Wiki}

El número de acciones GLAM Wiki que se llevan a cabo en España en los últimos años ha ido aumentado progresivamente, conforme se han ido difundiendo este tipo de actividades, y diferentes colectivos profesionales y de usuarios han entrado en contacto con ellas, principalmente a través de la participación activa. De entre todas ellas, se enumeran a continuación varias de ellas, en cuanto sirven para ejemplificar lo señalado en el apartado anterior.

- Fundación Joaquín Díaz: en 2013 se inició un proyecto de liberación de contenido entre la citada Fundación y Wikimedia España, que resultó en la liberación de más de 2700 fotografías antiguas de personas, lugares, monumentos y paisajes españoles (fig. 3). En 2015, esta liberación se amplió con más de 21000 archivos de audio de canciones y romances populares de distintas provincias de España, configurando uno de los mayores depósitos de este documentos sonoros para el estudio etnográfico español (Véase https://commons.wikimedia. org/wiki/Commons:Fundaci\%C3\%B3n_Joaqu\%C3\%ADn_D\%C3\%ADaz)

Fig. 3. Digitalización de foto antigua de la Fundación Joaquín Díaz (https://commons.wikimedia.org/wiki/ File:Fundaci\%C3\%B3n_Joaqu\%C3\%ADn_D\%C3\%ADaz_Acueducto_-_Segovia_(8).jpg)

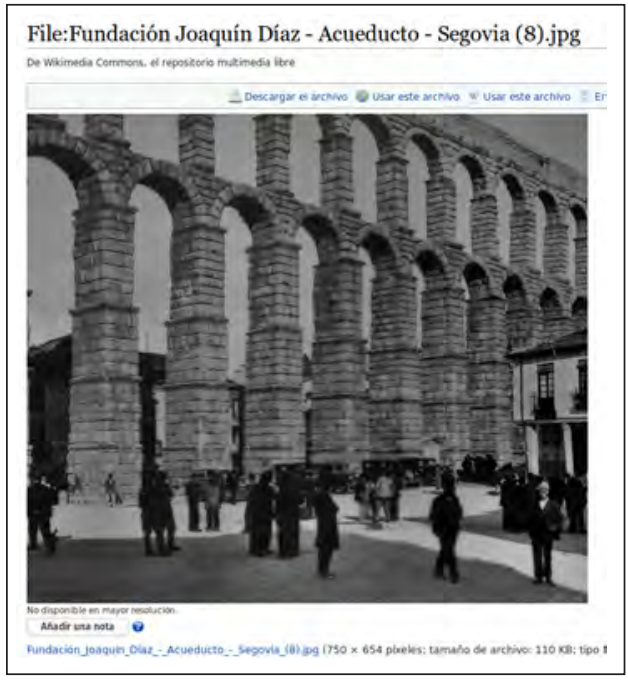

- GLAMing Madrid: se trata de un proyecto integrado, llevado a cabo por un wikipedista residente, entre septiembre de 2015 y enero de 2016, merced al convenio de colaboración firmado entre el Ministerio de Educación, Cultura y Deporte y Wikimedia España, y que tiene como objetivo la realización de actividades en colaboración con los Museos Estatales (Véase https:// meta.wikimedia.org/wiki/Grants:PEG/WM_ES/GLAMing_Madrid). El proyecto se desarrolló coordinadamente en el Museo Arqueológico Nacional, el Museo del Romanticismo y el Museo del Traje, y dentro de mismo se realizaron cursos de formación para el personal técnico de los citados museos, así como talleres de edición para los usuarios y visitantes de los mismos. Los museos procedieron a liberar digitalizaciones de elementos seleccionados de sus fondos en Commons, que han pasado a estar disponibles como dominio público. Como complemento al trabajo diario, se celebró un concurso de edición, el GLAMing Madrid Challenge, de un mes de duración, para la creación de artículos sobre contenidos de los museos, por una parte, y sobre mujeres célebres que hubieran estado vinculadas a estos museos, por otra. Además, tuvo lugar un editatón, el 17 de octubre de 2015, gracias al cual se mejoraron contenidos de los tres museos en Wikipedia. Como colofón, se grabaron dos conciertos de música del Romanticismo en este museo, que fueron liberados como dominio público en Commons con el permiso de los intérpretes (Ojeda y Tramullas, en prensa). 
- Wiki Takes Caesaraugusta: se trató de una actividad desarrollada durante un mañana, en septiembre de 2016, y en el ámbito de los museos municipales de Zaragoza que forman parte de la ruta de Caesaraugusta: los museos del Foro, del Puerto Fluvial, de la Termas Públicas y del Teatro. Se organizó por Wikimedia España y contó con el patrocinio del Ayuntamiento de Zaragoza. El objetivo del mismo fue documentar con amplitud y detalle los museos, sus estructuras y los contenidos expuestos en ellos, ya que su presencia en Wikipedia y Commons era escasa. Se obtuvieron y liberaron casi 500 fotografías de buena y alta calidad (fig. 4), que posteriomente se han usado para ilustrar artículos de Wikipedia (Véase https://commons. wikimedia.org/wiki/Category:Wiki_Takes_Caesaraugusta).

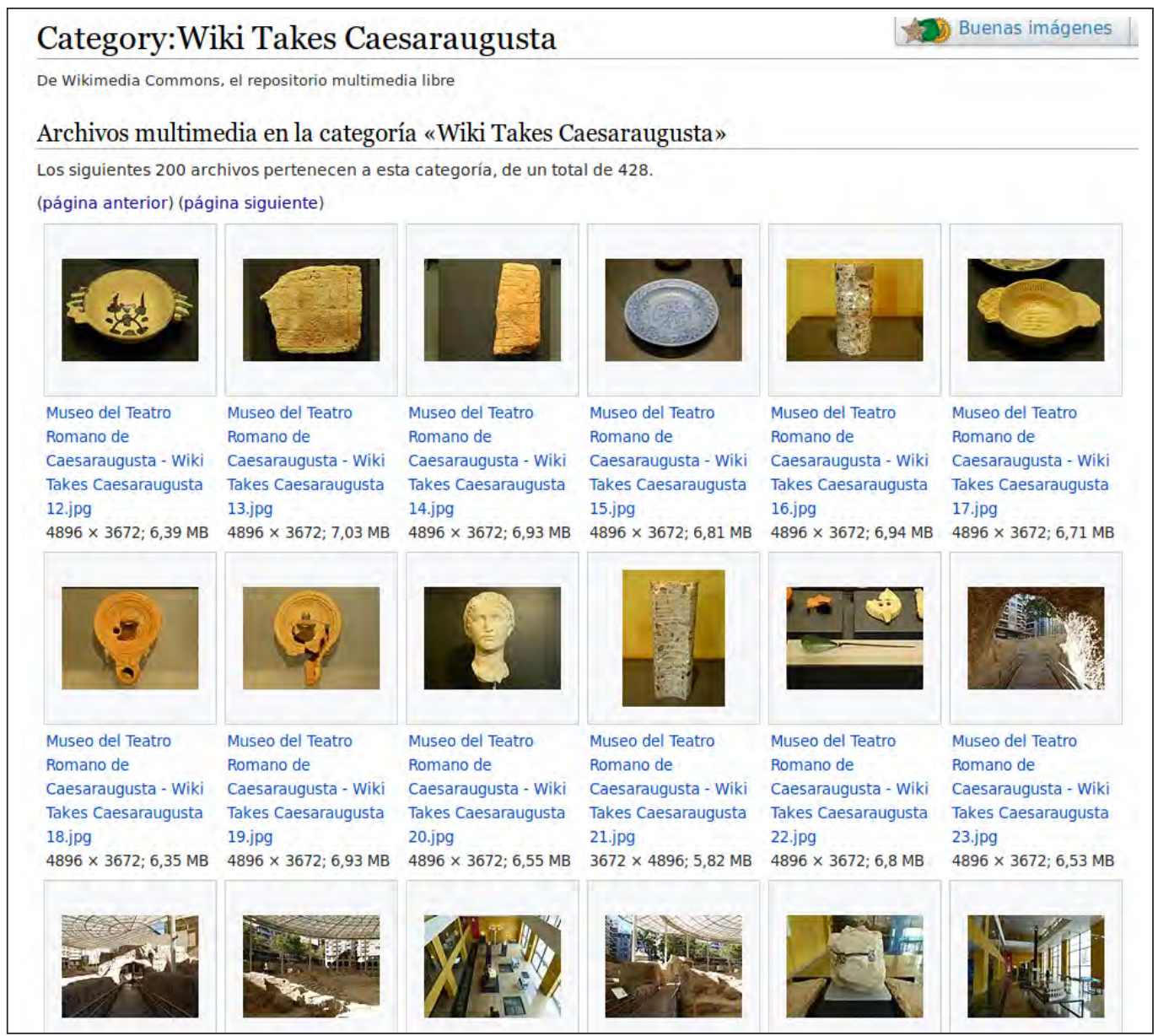

Fig. 4. Algunas de las fotografías obtenidas en Wiki Takes Caesaraugusta (https://commons.wikimedia.org/w/ index .php?title=Category:Wiki_Takes_Caesaraugusta\&filefrom=Museo+del+Teatro+Romano+de+Caesaraugusta++Wiki+Takes+Caesaraugusta+12.jpg\#mw-category-media)

- Wiki Takes Manchuela: en este caso la actividad se organizó como una ruta en una comarca, La Manchuela, en Albacete. Se trata de una comarca poco documentada, con un número de Lugares de Importancia Comunitaria (LIC), Bienes de Interés Cultural (BIC) y fiestas populares (Véase https://commons.wikimedia.org/wiki/Category:Wiki_Takes_La_Manchuela_2017). 
Durante dos días, en abril de 2017, un grupo de editores documentó gráficamente la arquitectura local, los espacios urbanos, lavaderos, cooperativas, patrimonio industrial, elementos de arqueología industrial, redes ferroviarias, centrales eléctricas... hasta alcanzar la cifra de más de 3000 fotografías. La mayor parte de ello no estaba recogido previamente, y ahora ha sido documentado y liberado en dominio público para su estudio y uso posterior por investigadores o cualquier otro tipo de usuario.

- Sorolla Challenge: se trata de un concurso específico organizado conjuntamente por Wikimedia España y el Museo Sorolla, durante el mes de noviembre de 2018, con la finalidad de aumentar el número de artículos en Wikipedia sobre las obras que conforman la colección del museo (Véase https://meta.wikimedia.org/wiki/Sorolla_Challenge/es). El concurso se hizo para cualquiera de las diferentes Wikipedias, lo que supuso creación de artículos en español, inglés, francés, alemán, catalán, neerlandés e incluso bengalí. Para facilitar la creación de contenido, el museo liberó en Commons reproducciones digitales de obras de su catálogo, con la finalidad de que ilustrasen los artículos creados.

\section{Conclusiones y perspectivas de futuro}

Las actuaciones dentro del contexto GLAM wiki se han perfilado en la última década como una de las mejores vías para la participación conjunta de las instituciones culturales y de los ciudadanos en el conocimiento y puesta en valor del patrimonio. Grandes instituciones culturales están llevando a cabo iniciativas de este tipo, con la seguridad de que el trabajo colaborativo es una de las vías que aseguran su perduración. La experiencia acumulada en esta clase de acciones permite delinear varias consideraciones:

- Es imprescindible e ineludible documentar, de forma digital, el patrimonio cultural. Esta es la única vía para asegurar la preservación del acervo y la memoria colectiva de las sociedades. Sólo la documentación previa puede permitir recuperar, al menos parcialmente, el patrimonio que desaparece o es destruido.

- La documentación se lleva a cabo para resolver problemas de acceso, uso y aprovechamiento. Wikimedia Commons es en la actualidad uno de los mayores repositorios mundiales de documentación fotográfica sobre patrimonio cultural, cuyo contenido es provisto de manera continuada por editores voluntarios y por instituciones. El uso de licencias libres permite el reaprovechamiento del material sin límites.

- La comunicación y difusión del patrimonio se revela como clave en las tareas de concienciar a las sociedades y de aumentar la relevancia de las instituciones culturales. La dominancia de la información visual en la actualidad obliga a desenvolverse en contexto en los cuales la imagen es fundamental; esto hace que las acciones de GLAM wiki sean la mejor vía para difundir el patrimonio.

- La capacidad para medir el impacto de las acciones GLAM wiki es una de las ventajas. En este entorno todo el mensurable: las aportaciones, los usos, el número de usuarios... lo que supone una ventaja notable cuando hay que realizar una rendición de cuentas y demostrar la rentabilidad del esfuerzo llevado a cabo sobre el patrimonio cultural.

El desarrollo colaborativo de a cciones sobre el patrimonio artístico y cultural es una aproximación que no puede desdeñarse (Oomen y Aroyo, 2011). Los recursos destinados a la cultura son decrecientes, y en muchas ocasiones implican una estructura de costes de difícil justificación, como demuestran los resultados de procesos de digitalización. Como acertadamente ha señalado Saorín (2013:82): “Nuestra sociedad no puede permitirse que los recursos públicos no estén donde hacen falta, y que se malgasten recursos en proyectos digitales de corto recorrido." Ante esta tesitura, la evolución, crecimiento y 
preservación del procomún requieren que los actores institucionales del panorama cultural sean conscientes, en primer lugar, de que son depositarios, que no propietarios, del patrimonio de la sociedad; en segundo, que sólo mediante la colaboración basada en el equilibrio entre las partes y sus aportaciones puede asegurar el disfrute y preservación del patrimonio cultural. Encerrar el patrimonio en una torre de cristal, aunque sea institucional, es seguir un camino hacia la irrelevancia.

\section{Bibliografía}

BOROWIECKI, Karol J., Trilce NAVARRETE (2017). "Digitization of heritage collections as indicator of innovation.", Economics of Innovation and New Technology, 26, 3, 227-246, doi: 10.1080/10438599.2016.1164488

COMISIÓN EUROPEA (2011). Recomendación de la Comisión de 27 de octubre de 2011 sobre la digitalización y accesibilidad en línea del material cultural y la conservación digital, Diario Oficial de la Unión Europea, 2011, L283/3045 [consulta: 6 septiembre 2018], disponible en:

http://eur-lex.europa.eu/LexUriServ/LexUriServ.do?uri=OJ:L:2011:283:0039:0045:ES:PDF

KAPSALIS, Effie (2016), The Impact of Open Access on Galleries, Libraries, Museums, \& Archives, Smithsonian Institution [consulta: 3 octubre 2017], disponible en: http://siarchives.si.edu/sites/default/files/pdfs/2016_03_10_ OpenCollections_Public.pdf

MACHEFERT, Sylvain (2015), "Improving the articles about modern art in Wikipedia: A partnership between Wikimédia France and the Pompidou Centre." Art Libraries Journal, 40, 3, 34-40, doi:10.1017/S030747220000033X

NAVARRETE, Trilce, Karol J. BOROWIECKI (2016), "Changes in cultural consumption: ethnographic collections in Wikipedia.", Cultural Trends, 25, 4, 233-248, doi: 10.1080/09548963.2016.1241342

OJEDA, Rubén, Jesús TRAMULLAS, (en prensa), "Líneas para el desarrollo de proyectos con Wikipedia y Wikimedia Commons en museos y bibliotecas.", En: IV Jornadas sobre Bibliotecas de Museos BIMUS, Madrid, 2017.

OJEDA, Rubén, WIKIMEDIA ESPAÑA (2016), Proyecto GLAMing Madrid, [consulta: 8 septiembre 2018], Disponible en: https://commons.wikimedia.org/wiki/File:Memoria_GLAMing_Madrid-es.pdf

OOMEN, Johan, Lora AROYO (2011), "Crowdsourcing in the cultural heritage domain.", En Proceedings of the 5th International Conference on Communities and Technologies - C\&T '11, New York: ACM Press, 138-149, doi:10.1145/2103354.2103373

PASTOR-SÁNCHEZ, Juan-Antonio, Tomás SAORÍN (2011), "Bancos de imágenes para proyectos enciclopédicos: el caso de Wikimedia Commons.", El profesional de la información, 20, 4, pp. 424-431, https://dx.doi.org/10.3145/ epi.2011.jul.09

PHILLIPS, Lory Bird (2013), "The Temple and the Bazaar: Wikipedia as a Platform for Open Authority in Museums.", Curator: The Museum Journal, 56, 2, 219-235, doi:10.1111/cura.12021

ROBICHAUD, Danielle (2016), "Wikipedia Edit-a-thons: Thinking Beyond the Warm Fuzzies.", Partnership: The Canadian Journal of Library and Information Practice and Research, 11, 2, doi:10.21083/partnership.v11i2.3802

SAORÍN, Tomás (2013), "Iniciativas GLAM-Wiki: Wikipedia como oportunidad para instituciones culturales.", Anuario ThinkEPI, 7, 78-85.

TERRAS, Melissa (2015), "Opening Access to collections: the making and using of open digitised cultural content.", Online Information Review, 39, 5, 733-752, [consulta: 14 octubre 2018], doi:10.1108/OIR-06-2015-0193

TUNCHS, Tomas (2007), "Museum Documentation and Wikipedia.de: Possibilities, opportunities and advantages for scholars and museums.", Museums and the Web 2007 [Consulta: 21 julio 2017], disponible en:

http://www.museumsandtheweb.com/mw2007/papers/tunsch/tunsch.html.

WIKIMEDIA ESPAÑA (2016), Introducción a los proyectos GLAM: cómo desarrollar una colaboración, [consulta: 22 octubre 2018], disponible en: https://commons.wikimedia.org/wiki/File:C\%C3\%B3mo_desarrollar_una_ colaboraci\%C3\%B3n_GLAM.pdf 